\title{
Cross-cultural adaptation of the Schizophrenia Caregiver Questionnaire (SCQ) and the Caregiver Global Impression (CaGl) Scales in 11 languages
}

Diana Rofail ${ }^{1}$, Catherine Acquadro ${ }^{2}$, Cécile Izquierdo ${ }^{3}$, Antoine Regnault ${ }^{4 *}$ and Steven H. Zarit ${ }^{5}$

\begin{abstract}
Background: The Schizophrenia Caregiver Questionnaire (SCQ) was developed to provide a comprehensive view of caregivers' subjective experiences of the impacts of caring for someone with schizophrenia. The Caregiver Global Impression $(\mathrm{CaGl})$ scales were designed to assess their perception of the severity of the schizophrenia symptoms, of change in schizophrenia symptoms and in the experience of caring since the beginning of the study. The objectives of the study were to translate the SCQ and CaGI scales in 11 languages [French (Canada, France), English (Canada, UK, Australia), German (Germany), Italian (Italy), Spanish (Spain), Dutch (the Netherlands), Finnish (Finland), and Swedish (Sweden)], to present evidence that the translations capture the concepts of the original questionnaires and are well understood by caregivers of patients with schizophrenia in each target country.

Methods: The different language versions were developed using a standard or adjusted linguistic validation process fully complying with the International Society for Pharmacoeconomics and Outcomes Research (ISPOR) recommended procedures.

Results: Interviews were conducted with 55 caregivers of patients with schizophrenia from 10 countries representing the 11 different languages. Participants ranged in age from 28 to 84 years and had 5 to 16 years of education. Women represented $69.1 \%$ (38/55) of the sample. Fourteen out of the 32 items of the SCQ generated difficulties which were mostly of semantic origin (13 items). The translation of the CaGl scales did not raise any major difficulty. Only five out of the 55 caregivers had difficulty understanding the meaning of the translations of "degree" in the expressions "degree of change in experience of caring" and "degree of change in symptoms".

Conclusions: Translations of the SCQ and CaGl scales into 11 languages adequately captured the concepts in the original English versions of the questionnaires, thereby demonstrating the conceptual, semantic, and cultural equivalence of each translation.
\end{abstract}

Keywords: Schizophrenia, Caregiver, Clinical outcome assessment, Translation, Cross-cultural adaptation

\section{Background}

Schizophrenia is a severe mental illness affecting 0.3-0.7\% of the population worldwide [1], characterized by three domains of psychopathology, including negative symptoms (social withdrawal, lack of motivation and emotional reactivity), positive symptoms (hallucinations, delusions) and cognitive deficits (working memory,

\footnotetext{
* Correspondence: aregnault@mapigroup.com

${ }^{4}$ HEOR \& Strategic Market Access, Mapi, 27 rue de la Villette, 69003 Lyon, France

Full list of author information is available at the end of the article
}

attention executive function) [2]. It is considered a leading cause of disability [3].

With the transition in care from formal hospital based healthcare systems to outpatient and community services and informal caregivers, it is estimated that $50-90 \%$ of people with chronic psychiatric illness live with their families or friends $[4,5]$. Informal caregivers (defined as "a person who has significant responsibility for managing the well-being of a person diagnosed with schizophrenia in an unpaid capacity" [6]) provide an important service by reducing the need for formal care and the burden upon healthcare systems [7].

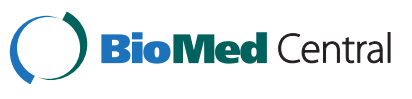

(c) 2015 Rofail et al.; licensee BioMed Central. This is an Open Access article distributed under the terms of the Creative Commons Attribution License (http://creativecommons.org/licenses/by/4.0), which permits unrestricted use, distribution, and reproduction in any medium, provided the original work is properly credited. The Creative Commons Public Domain Dedication waiver (http://creativecommons.org/publicdomain/zero/1.0/) applies to the data made available in this article, unless otherwise stated. 
The Schizophrenia Caregiver Questionnaire (SCQ) was developed to provide a comprehensive view of caregivers' subjective experiences of the impacts of caring for someone with schizophrenia [8]. It was adapted from the Zarit Burden Interview (ZBI) [9] in accordance with best practice recommendations for the development and modification of self-report measures [10]. The ZBI was originally developed to measure subjective burden among caregivers of adults with dementia. Items were generated based on clinical experience with caregivers and prior studies resulting in a 22-item self-report inventory that examines burden associated with functional/behavioural impairments and the home care situation. Based on findings from the literature, review of the ZBI, expert opinion, and faceto-face, semi-structured interviews with 19 US English speaking caregivers [11], changes were made to address concerns regarding relevance and sensitivity of the ZBI in the population of caregivers of patients with schizophrenia. The main changes consisted in the rewording of some items, the development of eleven additional items focusing on issues important to caregivers, the deletion of one item (i.e., item 9 of the ZBI - Do you feel strained when you are around your relative?), and the addition of a recall period (i.e., the past four weeks). In addition, the 5-point Likerttype response scale was replaced by an 11-point numerical rating scale (0-10 NRS). This change was decided with the hope that this modified response scale would allow more subtle changes to be captured by the questionnaire. The version of the SCQ that underwent cultural adaptation was a 32-item version, which was also the one that underwent psychometric validation.

The final version of the SCQ (after psychometric validation) is composed of 30 items covering 9 domains including: humanistic impact, exhaustion with caregiving role, lack of support, patient dependence, worries for the patient, perception of the care provided, financial dependence of the patient, financial impact of caregiving, overall difficulty of caregiving role.

In addition, a series of Caregiver Global Impression (CaGI) scales were also developed. These were designed for completion by caregivers to assess their perception of the severity of the schizophrenia symptoms of the person they care for over the past four weeks ["Please rate the severity of his/her symptoms during the past 4 weeks" scored "no symptoms" (0) to "very severe symptoms" (5)]; change in the schizophrenia symptoms of the person they care for since the beginning of the study ["Overall, how have his/her symptoms changed (if at all) since the beginning of the study (before starting treatment)?" scored from "very much improved" (1) to "very much worse" (7)]; and change in the experience of caring since the beginning of the study ["Overall, how much have your experiences of caring for a person with schizophrenia changed (if at all) since the beginning of the study (before the person started treatment)?" scored from "very much improved" (1) to "very much worse" (7)].

As for other conditions, clinical research in schizophrenia has become global [12]. In this context, outcome assessment has to be done in a multi-cultural framework; questionnaires need to be available in various languages to be included in multinational clinical trials and they need to appropriately capture the experience of individuals (patients or caregivers) with different linguistic and cultural backgrounds. Guidance for developing translations which are linguistically and culturally sound and respect the content validity of the original version has been developed by several organizations [13, 14]. In parallel, the Food and Drug Administration (FDA) requires that evidence about similarity of content validity and other measurement properties between the translated questionnaires and the original version be provided [10].

The objectives of the study were to prepare the SCQ and $\mathrm{CaGI}$ for use in a multicultural clinical research setting by conducting a proper translation of these instruments originally developed in US English in 11 languages, to present evidence that the translations capture the concepts of the original questionnaires and are well understood by caregivers of patients with schizophrenia in each target country. The translation of the SCQ was carried out on the instrument prior its psychometric validation (i.e., 32-item version).

\section{Methods}

\section{Linguistic validation process}

The instruments (SCQ and CaGI) were translated into languages that can be grouped into two "language families" (Indo-European and Uralic) based on their origins (Table 1).

The different language versions were developed using a standard or adjusted linguistic validation process fully complying with the International Society for Pharmacoeconomics and Outcomes Research (ISPOR) recommended procedures [13]. Fig. 1 illustrates the standard process. The conceptual analysis of the original questionnaires was performed with the developers of the original

Table 1 Language families and branches of the 11 target languages into which the SCQ and the CaGl scales were translated

\begin{tabular}{llll}
\hline $\begin{array}{l}\text { Language } \\
\text { family }\end{array}$ & Branch & & Language (country) \\
\hline Indo-European & Germanic & West & $\begin{array}{l}\text { Dutch [DUT] (The Netherlands), } \\
\text { English [ENG] (Australia, Canada, UK) } \\
\text { German [GER] (Germany) }\end{array}$ \\
& Italic & Romance & $\begin{array}{l}\text { French [FRE] (Canada, France), Italian } \\
\text { [ITA] (Italy), Spanish [SPA] (Spain) }\end{array}$ \\
& Uralic & Finnic & Finnish [FIN] (Finland) \\
\hline
\end{tabular}




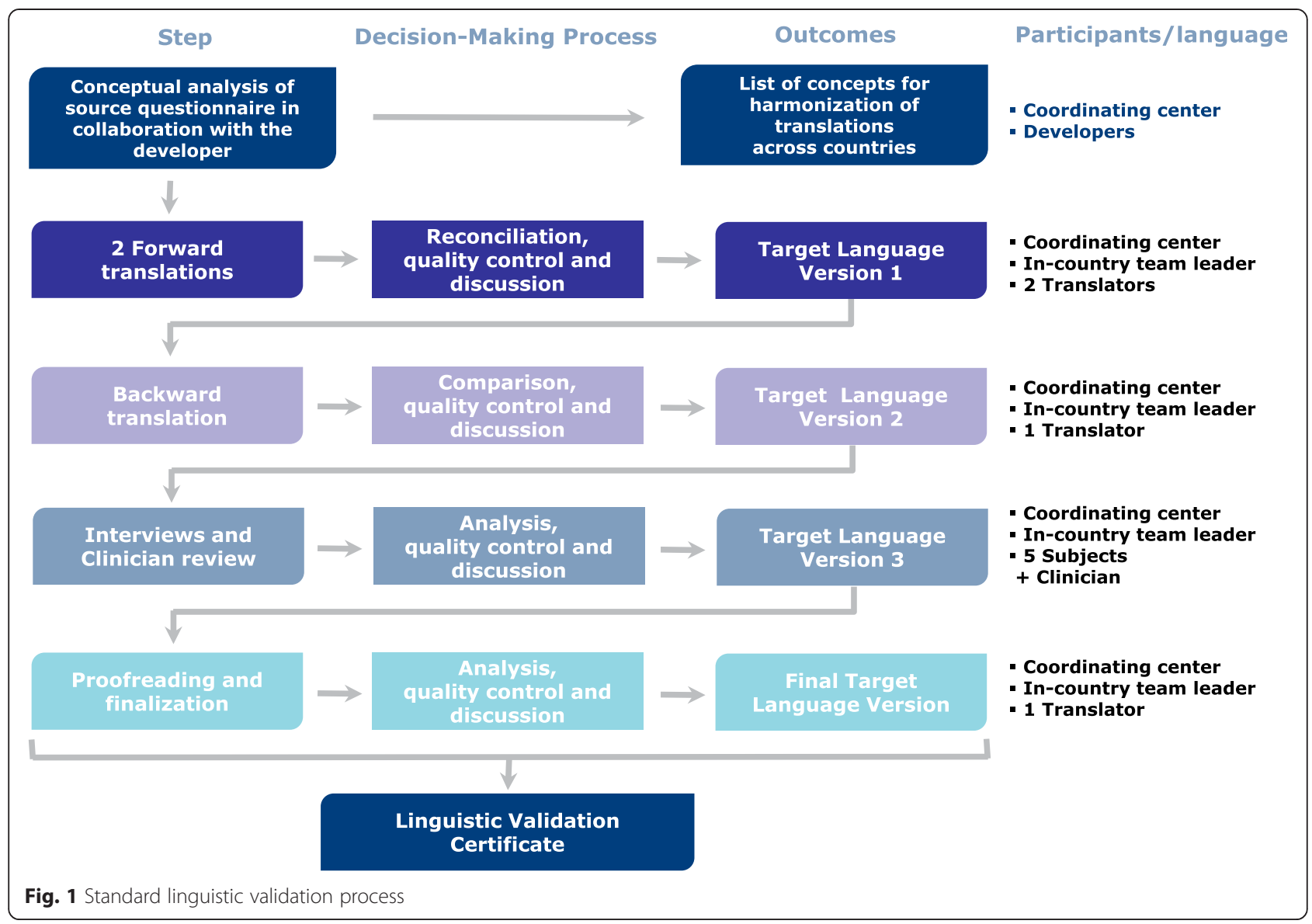

questionnaires in order to provide all translation teams with a document explaining the meaning of each item and suggesting terms to denote each concept. This was the basis for ensuring that all translations are harmonized between each other and faithful to the meaning of the original. Translations were developed by linguists through a process of dual forward translation (as per the ISPOR guidelines) and single back translation and reviews by a local team leader (linguist, psychologist or expert in survey research) and the project manager of the coordinating center who supervised all translations, in accordance with standards presented in Appendix A. The translations were then reviewed by a local clinician and tested via either telephone [French for Canada $(\mathrm{n}=5)$; English for Canada $(\mathrm{n}=$ $5)$, Finnish $(\mathrm{n}=2)$; Dutch $(\mathrm{n}=1)$ and English for Australia $(n=1)$ ] or face-to-face interviews of five caregivers of patients with schizophrenia living in the target countries. The convenience sample of 55 caregivers was recruited through the network of clinicians involved in the study. The interviews were conducted by the local team leader.

Language versions requiring the standard process were: French (Canada, France), German (Germany), Italian (Italy), Spanish (Spain), Dutch (the Netherlands), Finnish (Finland), and Swedish (Sweden).
For the adjusted process, the forward/backward step was replaced by an adaptation or localization step where an existing language version served as a basis for development of a separate local language version, e.g., US English version used as a basis to develop a UK English version. Language versions requiring an adjusted process were: English for Canada, UK, and Australia.

\section{Participants}

Participants of the interviews had to be native-speaking residents of the target countries and informal caregivers of patients with schizophrenia. Subject selection criteria for a sample of five participants per language were equal representation of gender, and representing people of mixed education (i.e., a minimum of two participants with less than 15 years of education).

In each country, caregivers were asked to complete the local language version of the SCQ and the CaGI, to indicate if they had any difficulties in understanding the instructions, the items, and response scales, and to paraphrase each sentence in the questionnaires or reformulate it with their own words. In each country, the interviewer carefully explored if the subjects had a clear understanding of the concept behind each item (Were they able to provide the 
meaning of each item?), and if not why (i.e., inaccurate translation or concept not culturally relevant?). The interviewer also checked the cultural relevance of the translation and asked for alternative wording if needed. For each instruction, item and response category, all difficulties and suggestions for changes, were gathered in a summary grid and analyzed to produce the final versions.

\section{Analysis}

All issues encountered for each questionnaire (SCQ, CaGI scales) during the full process and decisions made to solve them were summarized and were categorized as follows: Cultural (C), Idiomatic/Pragmatics (I), Semantic (S) and Syntactic (Sy). Table 2 illustrates each category.

\section{Results}

\section{Participants}

Interviews were conducted with 55 caregivers of patients with schizophrenia from 10 countries representing 11 different languages (see Table 1). The participants ranged in age from 28 to 84 years and had 5 to 16 years of education (Table 3 ). Caregivers were predominantly female (69\%),

Table 2 Categorization of translation difficulties

\begin{tabular}{ll}
\hline Category & Definition \\
\hline Cultural & A word or formulation in the original is culturally \\
& loaded in the target context due to societal or \\
& religious taboos (i.e., eating habits in Asian countries, \\
& taboos in Muslim areas). The usage of certain words \\
& or phrases based on the culture of a given society \\
& may be improper in the target language. \\
& E.g., starchy foods (e.g. potato, bread, etc.), starchy \\
foods (e.g. rice, pasta, chapatti, etc.).
\end{tabular}

Semantic Semantics concerns meanings, which are both denotative, i.e. the literal word (lexis), and connotative, namely the set of cultural and/or subjective associations implied by a word in addition to its literal explicit meaning. This category includes lexical differences and phraseology.

E.g., meet your responsibilities, meet your duties, meet your obligations.

Idiomatic/ Pragmatics

The practicalities of how a language is used in its everyday context are different between the source and target language. For example, one language may have more social registers than another (there are a number of different forms of addressing a person in Japanese, whereas English may only have one) and the idiosyncrasies of one language (repetitions, focus on particular words, etc.) may not be found in another.

E.g., I feel downhearted and blue, I feel down and sad.

Syntactic Correspond to specific aspects related to word morphology, sentence structure, grammar, punctuation. The structure and grammar of the source and target language diverge. For example, there is no grammatical form for the past tense in Tagalog.

E.g., How flexible have you been finding...? How flexible have you found...?
Table 3 Demographic characteristics of the caregivers* by target country

\begin{tabular}{llll}
\hline Country (language) & $\begin{array}{l}\text { Age } \\
\text { (years) }\end{array}$ & $\begin{array}{l}\text { Gender } \\
\text { (Males/Females) }\end{array}$ & $\begin{array}{l}\text { Education } \\
\text { (years) }\end{array}$ \\
\hline Australia (English) & $45-68$ & $1 / 4$ & $10-12$ \\
Canada (English) & $28-52$ & $2 / 3$ & $11-14$ \\
Canada (French) & $37-47$ & $2 / 3$ & $13-16$ \\
Finland (Finnish) & $35-66$ & $2 / 3$ & 12 \\
France (French) & $54-71$ & $2 / 3$ & $9-12$ \\
Germany (German) & $37-80$ & $1 / 4$ & $10-13$ \\
Italy (Italian) & $35-67$ & $2 / 3$ & $5-13$ \\
The Netherlands & $31-55$ & $1 / 4$ & $11-14$ \\
(Dutch) & $39-61$ & $0 / 5$ & $6-10$ \\
Spain (Spanish) & $40-60$ & $2 / 3$ & 9 \\
Sweden (Swedish) & $64-84$ & $2 / 3$ & $9-14$ \\
UK (English) & & & \\
\hline
\end{tabular}

${ }^{*} n=5$ for each language

which is consistent with previous research in samples of caregivers of patients with schizophrenia [15-17].

\section{SCQ}

Table 4 provides a summary of all difficulties that emerged during the translation process, in particular at the forward/backward translation steps and during the interviews. Fourteen out of the 32 items generated difficulties that raised discussions between the translators, the local team leader, and the central project manager. When an agreement could not be reached, the developer was consulted and gave the final decision. The difficulties were mostly of semantic origin (13 items). For instance, the meaning of "episode" (item 27) was found unclear or too technical by the caregivers and had to be qualified (i.e., psychotic, schizophrenic) or replaced by another word (i.e., crisis, relapse) to improve its clarity. The literal translation of "frustrated" (item 5) was not always possible and was replaced by the closest equivalent in the same semantic field. The only idiomatic expression present in the original version (i.e., "emotional rollercoaster" in item 31) was deleted in the languages of romance origin since it was not possible to find an idiomatic equivalent and the literal translation sounded too awkward (e.g., "montagne russe émotionnelle" in French).

The various types of changes that were required are summarized in Table 4.

The clinician review did not reveal any problematic issue before the test with the caregivers.

An overview of the comprehension rate, language by language and item by item, is provided in Table 5 . The SCQ was globally well-understood by most of the respondents (overall $=98.86 \%$ ).

On the conceptual level, Anglophone caregivers in Canada argued about the relevance of item 26 (Over the 
Table 4 Most problematic SCQ items classified by type of difficulty (Diff.) - Cultural (C), Idiomatic/Pragmatics (I), Semantic (S) and Syntactic (Sy)

Item Diff. Lang.* (country) LV Step Description

\section{F/B IS}

15. Over the past four weeks, how often did you C ENG (Canada) feel that you didn't have enough money to care

for him/her, in addition to the rest of your expenses?

31. Over the past four weeks, how often did you I ENG (Canada), experience emotional highs and lows ("an emotional rollercoaster") because of his/her schizophrenia?

1. Over the past four weeks, how often did you feel that he/she asked for more help than needed?

3. Over the past four weeks, how difficult was it $S$ for you to care for him/her and meet your other responsibilities?

4. Over the past four weeks, how embarrassed did you feel about his/her behavior?

5. Over the past four weeks, how frustrated did S you feel about his/her behavior?

FRE (France)

11. Over the past four weeks, how often did you $\mathrm{S}$ feel that you didn't have as much privacy as you would have liked because of him/her?

14. Over the past four weeks, how often did you S feel that he/she was overly dependent on you to help with daily activities?

20. Over the past four weeks, how often did you S FRE (Canada) feel you should be doing more for him/her?

FRE (France),

FIN, ITA, SPA

SWE

FRE (Canada,

France), GER,

ITA, SPA

ITA, SPA

FRE (France) $\checkmark$ All respondents were somewhat confused by this item, since in Canada patients with schizophrenia automatically qualify for government assistance and their medications are covered by social insurance.

$\checkmark$ "High and lows" was translated either by a corresponding idiomatic expression or by a non idiomatic equivalent. translators and respondents of romance languages.

Meaning of "he/she" was found unclear by translators.

$\checkmark$ Meaning of "you" was considered too ambiguous by respondents.

$\checkmark$ "Embarrassed" was translated with a word meaning both "ashamed" and "embarrassed". The interviews suggested that the respondents gave it a meaning that had a strong connotation.

$\checkmark$ Two respondents were disturbed by the translation of embarrassed ("ashamed").

$\checkmark$ The literal translation of frustrated was not understood by some respondents.

The literal translation of frustrated was not possible. It was translated by an equivalent of "irritated". The interviews a strong connotation.

$\checkmark$ Respondents argued that the Dutch word did not convey the meaning of "time for oneself" and suggested another expression.

The word "privacy" in French could also mean "intimacy".

$\checkmark$ The meaning of "overly" was not clear for some $\checkmark \quad \checkmark$ "Emotional rollercoaster" was perceived as too idiomatic by suggested that the respondents gave it a meaning that had respondents.

$\checkmark$ Item well understood. However the respondents found that "Doing more" was underlined in the translation its meaning was too close to the meaning of item 21.
Item reworded as follows: "Over the past four weeks, how often did you feel that, in addition to the rest of your expenses, you needed more money to care for him/her?"

ENG, FRE (France): "ups and down", FIN, ITA, SPA: "mood swings", SWE: "valleys and tops"

FRE (Canada), ITA, SPA: deleted, FRE (France): "up and down morale", GER: "rollercoaster feelings""

"He/she" translated by "ill person" (Italian) [changed made for all items including he/she], "the person you take care of" (Spanish)

"You" was removed, "him/her" replaced by "ill person". Change made for all items with him/her

Replaced by a more direct equivalent of "embarrassed"

No change

Translated by an equivalent of "dissatisfied"

"Irritated" replaced by an equivalent of "annoyed" "Private life"

\section{No change}

"Overly" replaced by an equivalent of "too" 
Table 4 Most problematic SCQ items classified by type of difficulty (Diff.) - Cultural (C), Idiomatic/Pragmatics (I), Semantic (S) and Syntactic (Sy) (Continued)

27. Over the past four weeks, how often did you worry that he/she might have an episode?

\section{Over the past four weeks, how often did you} feel that his/her schizophrenia affected your

relationship with other family members or

friends in a negative way?

17. Over the past four weeks, how often did you feel you weren't in control of your life because of his/her schizophrenia?

28. Over the past four weeks, how often did you worry that his/her schizophrenia might get worse?

31. Over the past four weeks, how often did you experience emotional highs and lows ("an

emotional rollercoaster") because of his/her schizophrenia?

8. Over the past four weeks, how afraid were you sy of what the future holds for him/her?
FRE (Canada)

ENG (Canada), ITA, GER, SPA, SWE

\section{ENG (Canada,}

UK), FRE

(Canada)

Sy ITA an unwanted ambiguity: "your" is also translated as "his/

her", i.e., "your relationship" may be understood as "his/her

relationship", "his/her schizophrenia" as "your schizophrenia".

$\checkmark$ "Episode" was either perceived unclear or too technical by respondents.

$\checkmark$ Items well understood. However some respondents were strongly opposed to the use of "schizophrenia" because of the stigma attached to it.
Addition of "psychotic" to clarify meaning

ENG: "psychotic break", "GER: "schizophrenic episode", ITA SPA: "crisis", SWE: "relapse"

\section{No change [decision of the developer]}

"His/her" was deleted in the Italian version

$\checkmark$ The item was well understood but the respondents found "How worried were you about his/her future" its structure unnecessarily too complicated.

* Lang.: Language; ${ }^{\S}$ LV: Linguistic Validation; F/B: issues discussed at forward/backward steps; IS: issues discussed during interviews step 
Table $\mathbf{5}$ SCQ comprehension rates by languages and by items during interviews*

\begin{tabular}{|c|c|c|c|c|c|c|c|c|c|c|c|c|c|}
\hline Items & DUT & $\begin{array}{l}\text { ENG } \\
(\mathrm{Au})\end{array}$ & $\begin{array}{l}\text { ENG } \\
\text { (Can) }\end{array}$ & $\begin{array}{l}\text { ENG } \\
(\text { UK) }\end{array}$ & $\begin{array}{l}\text { FRE } \\
\text { (Can) }\end{array}$ & $\begin{array}{l}\text { FRE } \\
\text { (Fran) } \\
\end{array}$ & FIN & GER & ITA & SPA & SWE & $\begin{array}{l}\text { Total by } \\
\text { Item }\end{array}$ & \\
\hline 1 & $4 / 5$ & $5 / 5$ & $5 / 5$ & $5 / 5$ & $5 / 5$ & $5 / 5$ & $5 / 5$ & $5 / 5$ & $5 / 5$ & $5 / 5$ & $5 / 5$ & $54 / 55$ & $98.18 \%$ \\
\hline 2 & $5 / 5$ & $5 / 5$ & $5 / 5$ & $5 / 5$ & $5 / 5$ & $5 / 5$ & $5 / 5$ & $5 / 5$ & $5 / 5$ & $5 / 5$ & $5 / 5$ & $55 / 55$ & $100 \%$ \\
\hline 3 & $5 / 5$ & $5 / 5$ & $5 / 5$ & $5 / 5$ & $5 / 5$ & $5 / 5$ & $5 / 5$ & $5 / 5$ & $3 / 5$ & $5 / 5$ & $5 / 5$ & $53 / 55$ & $96.36 \%$ \\
\hline 4 & $5 / 5$ & $5 / 5$ & $5 / 5$ & $5 / 5$ & $5 / 5$ & $5 / 5$ & $5 / 5$ & $3 / 5$ & $5 / 5$ & $5 / 5$ & $5 / 5$ & $53 / 55$ & $96.36 \%$ \\
\hline 5 & $5 / 5$ & $5 / 5$ & $5 / 5$ & $5 / 5$ & $5 / 5$ & $5 / 5$ & $5 / 5$ & $5 / 5$ & $5 / 5$ & $5 / 5$ & $5 / 5$ & $55 / 55$ & $100 \%$ \\
\hline 6 & $3 / 5$ & $5 / 5$ & $5 / 5$ & $5 / 5$ & $5 / 5$ & $5 / 5$ & $5 / 5$ & $5 / 5$ & $5 / 5$ & $5 / 5$ & $5 / 5$ & $53 / 55$ & $96.36 \%$ \\
\hline 7 & $4 / 5$ & $5 / 5$ & $5 / 5$ & $5 / 5$ & $5 / 5$ & $5 / 5$ & $5 / 5$ & $5 / 5$ & $5 / 5$ & $5 / 5$ & $5 / 5$ & $54 / 55$ & $98.18 \%$ \\
\hline 8 & $5 / 5$ & $5 / 5$ & $5 / 5$ & $5 / 5$ & $5 / 5$ & $5 / 5$ & $5 / 5$ & $5 / 5$ & $5 / 5$ & $5 / 5$ & $5 / 5$ & $55 / 55$ & $100 \%$ \\
\hline 9 & $5 / 5$ & $5 / 5$ & $5 / 5$ & $5 / 5$ & $5 / 5$ & $5 / 5$ & $5 / 5$ & $5 / 5$ & $5 / 5$ & $5 / 5$ & $5 / 5$ & $55 / 55$ & $100 \%$ \\
\hline 10 & $5 / 5$ & $5 / 5$ & $5 / 5$ & $5 / 5$ & $5 / 5$ & $5 / 5$ & $5 / 5$ & $5 / 5$ & $4 / 5$ & $5 / 5$ & $5 / 5$ & $54 / 55$ & $98.18 \%$ \\
\hline 11 & $5 / 5$ & $4 / 5$ & $5 / 5$ & $5 / 5$ & $5 / 5$ & $5 / 5$ & $5 / 5$ & $5 / 5$ & $5 / 5$ & $5 / 5$ & $5 / 5$ & $54 / 55$ & $98.18 \%$ \\
\hline 12 & $5 / 5$ & $5 / 5$ & $5 / 5$ & $5 / 5$ & $5 / 5$ & $5 / 5$ & $5 / 5$ & $5 / 5$ & $5 / 5$ & $5 / 5$ & $5 / 5$ & $55 / 55$ & $100 \%$ \\
\hline 13 & $5 / 5$ & $5 / 5$ & $5 / 5$ & $5 / 5$ & $5 / 5$ & $5 / 5$ & $5 / 5$ & $5 / 5$ & $5 / 5$ & $5 / 5$ & $5 / 5$ & $55 / 55$ & $100 \%$ \\
\hline 14 & $3 / 5$ & $5 / 5$ & $5 / 5$ & $5 / 5$ & $5 / 5$ & $5 / 5$ & $5 / 5$ & $5 / 5$ & $5 / 5$ & $5 / 5$ & $5 / 5$ & $53 / 55$ & $96.36 \%$ \\
\hline 15 & $5 / 5$ & $5 / 5$ & $5 / 5$ & $5 / 5$ & $5 / 5$ & $5 / 5$ & $5 / 5$ & $5 / 5$ & $5 / 5$ & $5 / 5$ & $5 / 5$ & $55 / 55$ & $100 \%$ \\
\hline 16 & $5 / 5$ & $5 / 5$ & $5 / 5$ & $5 / 5$ & $5 / 5$ & $5 / 5$ & $5 / 5$ & $5 / 5$ & $4 / 5$ & $5 / 5$ & $5 / 5$ & $54 / 55$ & $98.18 \%$ \\
\hline 17 & $5 / 5$ & $5 / 5$ & $5 / 5$ & $5 / 5$ & $5 / 5$ & $5 / 5$ & $5 / 5$ & $5 / 5$ & $5 / 5$ & $5 / 5$ & $5 / 5$ & $55 / 55$ & $100 \%$ \\
\hline 18 & $5 / 5$ & $5 / 5$ & $5 / 5$ & $5 / 5$ & $5 / 5$ & $5 / 5$ & $5 / 5$ & $5 / 5$ & $5 / 5$ & $5 / 5$ & $5 / 5$ & $55 / 55$ & $100 \%$ \\
\hline 19 & $5 / 5$ & $4 / 5$ & $5 / 5$ & $5 / 5$ & $5 / 5$ & $5 / 5$ & $5 / 5$ & $5 / 5$ & $5 / 5$ & $5 / 5$ & $5 / 5$ & $54 / 55$ & $98.18 \%$ \\
\hline 20 & $5 / 5$ & $5 / 5$ & $5 / 5$ & $5 / 5$ & $5 / 5$ & $5 / 5$ & $5 / 5$ & $5 / 5$ & $5 / 5$ & $5 / 5$ & $5 / 5$ & $55 / 55$ & $100 \%$ \\
\hline 21 & $5 / 5$ & $5 / 5$ & $5 / 5$ & $5 / 5$ & $5 / 5$ & $5 / 5$ & $5 / 5$ & $5 / 5$ & $5 / 5$ & $5 / 5$ & $5 / 5$ & $55 / 55$ & $100 \%$ \\
\hline 22 & $5 / 5$ & $5 / 5$ & $4 / 5$ & $5 / 5$ & $5 / 5$ & $5 / 5$ & $5 / 5$ & $5 / 5$ & $5 / 5$ & $5 / 5$ & $5 / 5$ & $54 / 55$ & $98.18 \%$ \\
\hline 23 & $5 / 5$ & $5 / 5$ & $5 / 5$ & $5 / 5$ & $5 / 5$ & $5 / 5$ & $5 / 5$ & $5 / 5$ & $5 / 5$ & $5 / 5$ & $5 / 5$ & $55 / 55$ & $100 \%$ \\
\hline 24 & $5 / 5$ & $5 / 5$ & $5 / 5$ & $5 / 5$ & $5 / 5$ & $5 / 5$ & $5 / 5$ & $5 / 5$ & $5 / 5$ & $5 / 5$ & $5 / 5$ & $55 / 55$ & $100 \%$ \\
\hline 25 & $5 / 5$ & $5 / 5$ & $5 / 5$ & $5 / 5$ & $5 / 5$ & $5 / 5$ & $5 / 5$ & $5 / 5$ & $5 / 5$ & $5 / 5$ & $5 / 5$ & $55 / 55$ & $100 \%$ \\
\hline 26 & $5 / 5$ & $5 / 5$ & $5 / 5$ & $5 / 5$ & $5 / 5$ & $5 / 5$ & $5 / 5$ & $5 / 5$ & $5 / 5$ & $5 / 5$ & $5 / 5$ & $55 / 55$ & $100 \%$ \\
\hline 27 & $5 / 5$ & $5 / 5$ & $5 / 5$ & $5 / 5$ & $5 / 5$ & $5 / 5$ & $5 / 5$ & $5 / 5$ & $5 / 5$ & $5 / 5$ & $5 / 5$ & $55 / 55$ & $100 \%$ \\
\hline 28 & $5 / 5$ & $5 / 5$ & $5 / 5$ & $5 / 5$ & $5 / 5$ & $5 / 5$ & $5 / 5$ & $5 / 5$ & $5 / 5$ & $5 / 5$ & $5 / 5$ & $55 / 55$ & $100 \%$ \\
\hline 29 & $5 / 5$ & $5 / 5$ & $4 / 5$ & $5 / 5$ & $5 / 5$ & $5 / 5$ & $5 / 5$ & $5 / 5$ & $5 / 5$ & $5 / 5$ & $5 / 5$ & $54 / 55$ & $98.18 \%$ \\
\hline 30 & $5 / 5$ & $5 / 5$ & $5 / 5$ & $5 / 5$ & $5 / 5$ & $5 / 5$ & $5 / 5$ & $5 / 5$ & $5 / 5$ & $5 / 5$ & $5 / 5$ & $55 / 55$ & $100 \%$ \\
\hline 31 & $5 / 5$ & $5 / 5$ & $5 / 5$ & $5 / 5$ & $5 / 5$ & $5 / 5$ & $5 / 5$ & $5 / 5$ & $5 / 5$ & $5 / 5$ & $2 / 5$ & $52 / 55$ & $94.54 \%$ \\
\hline 32 & $5 / 5$ & $4 / 5$ & $5 / 5$ & $5 / 5$ & $5 / 5$ & $5 / 5$ & $5 / 5$ & $5 / 5$ & $5 / 5$ & $5 / 5$ & $5 / 5$ & $54 / 55$ & $98.18 \%$ \\
\hline \multirow[t]{2}{*}{$\begin{array}{l}\text { Total by } \\
\text { language }\end{array}$} & $\begin{array}{l}154 / \\
160\end{array}$ & $\begin{array}{l}157 / \\
160\end{array}$ & $158 / 160$ & $\begin{array}{l}160 / \\
160\end{array}$ & $\begin{array}{l}160 / \\
160\end{array}$ & $\begin{array}{l}160 / \\
160\end{array}$ & $\begin{array}{l}160 / \\
160\end{array}$ & $\begin{array}{l}158 / \\
160\end{array}$ & $\begin{array}{l}156 / \\
160\end{array}$ & $\begin{array}{l}160 / \\
160\end{array}$ & $\begin{array}{l}157 / \\
160\end{array}$ & $\begin{array}{l}1740 / \\
1760\end{array}$ & $98.86 \%$ \\
\hline & $96.25 \%$ & $98.13 \%$ & $98.75 \%$ & $100 \%$ & $100 \%$ & $100 \%$ & $100 \%$ & $98.75 \%$ & $97.50 \%$ & $100 \%$ & $98.13 \%$ & $98.86 \%$ & \\
\hline
\end{tabular}

*5 caregivers in each country

past four weeks, how difficult was it to get him/her to take his/her medication?). They did not see the point of asking this question ("They assume it's a difficulty? That doesn't make sense. Not sure what this question is getting $\left.a t^{\prime \prime}\right)$. Other caregivers in Finland and the Netherlands pointed out that item 26 was not relevant in case of injections. Interestingly, this item was deleted following the outcomes of the psychometric validation.

\section{CaGl scales}

The translation of the CaGI scales in the 11 languages did not raise any major difficulty. Only five out of the 55 caregivers [one in Finland, four in Canada (two French, two English)] had difficulty understanding the meaning of the translations of "degree" in the expressions "degree of change in experience of caring" and "degree of change in symptoms". Issues were resolved by either deleting 
"degree of" [English (Canada), Finnish], or using the word "evolution of" to replace "degree of change" [French (Canada)]. The term "evolution" was chosen for its neutral connotation implying either a worsening or an improvement. The overall comprehension rate of the CaGI scales was $99.17 \%$.

\section{Discussion}

The results of the linguistic validation process indicate that the translations of the SCQ and the CaGI scales into 11 European languages adequately captured the concepts of the original English-language version of the questionnaires and were easily understood by caregivers of patients with schizophrenia. For each translation, it was important to utilize everyday language, yet remain true to the original meaning of the items. This involved focusing on semantics and required extensive discussion about the meaning of each concept and the adequate word to be chosen to convey it. This was accomplished through steady and continual discussion between the local team of translators, the local team leader, the central project manager and the developers of the original questionnaires. The initial conceptual analysis was crucial to obtaining translations harmonized with each other and faithful to the meaning of the original. The participation of caregivers of patients with schizophrenia greatly improved the initial translations, and provided input essential to the development of versions easily understood by the target population. In most cases, the solutions to each difficulty were decided collegially. Each decision was documented to enable further discussion at the time of the psychometric testing of the translations. The involvement of the caregivers in the development of the original version of the SCQ and its translations was essential. This is in the line with recent reviews which stress the need for tools with relevant content based on caregivers' views $[18,19]$.

Cross-cultural equivalence is a critical factor/an essential component for instruments used in multi-cultural studies. Demonstrating cross-cultural equivalence entails investigating that the instrument measures the same concepts in a similar way across the different languages and cultures [20,21]. It facilitates the pooling of data collected by the different languages versions of the instrument, and in the context of clinical trials, it optimizes the chance of demonstrating treatment benefit by improving the quality of measurement of the outcomes of interest [22]. Applying proper linguistic validation methodology contributes to the achievement of crossculturally equivalent versions of instruments by optimizing the different languages versions. In addition, this process can generate useful data to document crosscultural equivalence. During the linguistic validation of the SCQ and CaGI scales in 11 languages, no major cultural issues emerged. This shows that the concepts that were identified in the development of the original US English version of the questionnaire were relevant to the caregivers in other countries, supporting the existence of an overall common experience of the caregivers of patients with schizophrenia in a range of countries. This finding is important, given the recognized importance of cultural aspects in schizophrenia [23].

We have identified several limitations to our research: first, the use of a convenience sample (not representative of the entire population of caregivers of patients with schizophrenia), and the use of telephone interviews for some languages [French for Canada $(n=5)$; English for Canada $(\mathrm{n}=5)$, Finnish $(\mathrm{n}=2)$; Dutch $(\mathrm{n}=1)$ and English for Australia $(n=1)]$, which may have prevented the interviewer to capture all the subtleties of body language during the completion of the questionnaire, and finally the fact that this research was conducted in Western countries only. It would be of great interest to extend this cross-cultural research on the experience of caregivers of patients with schizophrenia in a wider spectrum of countries and cultures. A recent review has shown that sociocultural and ethnic characteristics play an important role in the perception of family caregivers' burden [24]. However, published research on cross-cultural perspectives in caring for someone with schizophrenia is still limited. A PubMed search with key words such as "schizophrenia," "cross-cultural" and "caregiver" showed that publications in this area represent only $4 \%(n=38)$ of the papers published in schizophrenia and cross-cultural research $(n=942)$. Most of the studies operate within one continent [25-27] or within relatively similar cultural settings (i.e., Chinese-speaking countries) [28]. Very few compare populations such as ethnic groups within one country $[29,30]$ or caregivers from very different geographical areas or levels of development [31]. In a study comparing the health-related quality of life of French and Chilean caregivers, Boyer et al. [31] use a generic questionnaire (i.e., the SF-36) and show similar levels of health-related quality of life.

The availability in 11 European languages of diseasespecific questionnaires such as the SCQ and the CaGI scales to assess the impact of caring for patients with schizophrenia is the first step to wider development and use in various cultural settings. International studies assessing differences of impact across cultures would be of great interest. They would enable cross-cultural comparisons, improve awareness, tracking, and management of impact on caregivers of patients with schizophrenia in different cultures, thus providing opportunity for increased support.

\section{Conclusions}

Translations of the Schizophrenia Caregiver Questionnaire and the Caregiver Global Impression scales into 11 
languages adequately captured the concepts in the original English version of the questionnaires, thereby demonstrating the conceptual, semantic, and cultural equivalence of each translation.

After psychometric testing, the instruments will be available for use in clinical trials facilitating international comparison and pooling of data and will provide new insights into the area of caregiving of schizophrenia.

\section{Appendix A. Translation procedures}

1. Two translators who are native speakers of the target language and are experienced in translating health questionnaires independently translate the document.

2. After both translations are complete, the local team leader compares the two translations and produces a third translation. If difficulties arise, discussions with both translators take place to reach agreement. This process of discussion and review is known as "reconciling" and the resulting third translation is referred to as the target language version \#1.

3. The translation is then given to a native Englishspeaking translator for translation back into English. This document is referred to as the "back-translation."

4. The local team leader and the project manager of the coordinating center (central project manager) compare the original English to the back-translation and either approve or question each item in the back-translation.

5. The central project manager discusses concerns with the local (in-country) team leader who may change the translation, change the back-translation, or leave the translation as it is, providing a justification to the central project manager.

6. This process is repeated until all translation issues have been resolved and the revised back-translation is approved. The resulting translation is the target language version \#2 which will be reviewed by a clinician and tested with subjects (interviews).

\footnotetext{
Abbreviations

C: Cultural; CaGl: Caregiver Global Impression; Diff: Difficulty; F/B: Forward/ backward step; FDA: Food and Drug Administration; I: Idiomatic/Pragmatics; IS: Interviews step; ISPOR: International Society for Pharmacoeconomics and Outcomes Research; Lang: Language; LV: Linguistic validation;

SCQ: Schizophrenia Caregiver Questionnaire; S: Semantic; SF-36: Short Form (36) Health Survey; Sy: Syntactic; US: United States; UK: United Kingdom; ZBI: Zarit Burden Interview.
}

\section{Competing interests}

The manuscript was financed by Roche Pharmaceuticals. DR is a full employee of Roche Pharmaceuticals. AR, CA and $\mathrm{Cl}$ are consultants paid by Roche Pharmaceuticals. SHZ and Roche Pharmaceuticals share the copyright of the SCQ (original and translations). SHZ may obtain royalties from any use of the SCQ and translations.

\section{Authors' contributions}

DR conceived, designed the project and helped to draft the manuscript. Cl coordinated the project and helped to draft the manuscript. CA and AR analyzed the data and drafted the manuscript. SHZ developed the original SCQ and reviewed the process of translation. All authors read and approved the final manuscript.

\section{Acknowledgements}

We are extremely grateful to the caregivers who have contributed to this study.

We would like to thank Carmen Berardo (formely Roche Pharmaceuticals, now Genentech Inc.) for her support and critique of the study.

\section{Author details}

${ }^{1}$ Patient-Centered Outcomes Research for Neuroscience \& Metabolism, Roche Products Ltd, Welwyn Garden City, UK. ' Mapi Research Trust, Lyon, France. ${ }^{3}$ Language Services, Mapi, Lyon, France. ${ }^{4}$ HEOR \& Strategic Market Access, Mapi, 27 rue de la Villette, 69003 Lyon, France. ${ }^{5}$ Department Head, Human Development and Family Studies, The Pennsylvania State University, University Park, PA, USA.

Received: 4 December 2014 Accepted: 15 May 2015

Published online: 09 June 2015

\section{References}

1. van Os J, Kapur S. Schizophrenia. Lancet. 2009;374:635-45

2. Tandon R, Nasrallah HA, Keshavan MS. Schizophrenia, just the facts 4. Clinical features and conceptualization. Schizophr Res. 2009;110:1-23.

3. Switaj P, Anczewska M, Chrostek A, Sabariego C, Cieza A, Bickenbach J, et al. Disability and schizophrenia: a systematic review of experienced psychosocial difficulties. BMC Psychiatry. 2012;12:193.

4. Rossler W, Salize HJ, Cucchiaro G, Reinhard I, Kernig C. Does the place of treatment influence the quality of life of schizophrenics? Acta Psychiatr Scand. 1999;100:142-8.

5. Honkonen T, Saarinen S, Salokangas RK. Deinstitutionalization and schizophrenia in Finland II: discharged patients and their psychosocial functioning. Schizophr Bull. 1999;25:543-51.

6. Nevada Revised Statutes (NRS) 453A.080. 2012 [http://definitions.uslegal.com/c/ caregiver/]

7. Arno PS, Levine C, Memmott MM. The economic value of informal caregiving Health Aff. 1999;18:182-8.

8. Adam Gater A, Rofail D, Marshall C, Abetz L, Zarit SH, Galani-Berardo C. Assessing the impact of caring for a person with schizophrenia: Development of the Schizophrenia Caregiver Questionnaire. Patient. 2015. doi:10.1007/s40271-015-0114-3.

9. Zarit SH, Reever KE, Bach-Peterson J. Relatives of the impaired elderly: correlates of feelings of burden. Gerontologist. 1980;20:649-55.

10. Food and Drug Administration. Patient-reported outcome measures: use in medical product development to support labeling claims. Fed Regist. 2009;74:65132-3. http://www.fda.gov/downloads/Drugs/Guidances/ UCM193282.pdf.

11. Gater A, Rofail D, Tolley C, Marshall C, Abetz-Webb L, Zarit SH, et al. "Sometimes It's Difficult to Have a Normal Life": Results from a Qualitative Study Exploring Caregiver Burden in Schizophrenia. Schizophr Res Treat. 2014;2014:368215.

12. Hong J, Novick D, Brugnoli R, Karagianis J, Dossenbach M, Haro JM. Clinical consequences of switching from olanzapine to risperidone and vice versa in outpatients with schizophrenia: 36 -month results from the Worldwide Schizophrenia Outpatients Health Outcomes (W-SOHO) study. BMC Psychiatry. 2012;12:218.

13. Wild D, Grove A, Martin M, Eremenco S, McElroy S, Verjee-Lorenz A, et al. Principles of Good Practice for the Translation and Cultural Adaptation Process for Patient-Reported Outcomes (PRO) Measures: report of the ISPOR Task Force for Translation and Cultural Adaptation. Value Health. 2005;8:94-104.

14. Acquadro C, Conway K, Giroudet C, Mear I. Linguistic validation manual for health outcome assessments. Lyon: Mapi Institute; 2012.

15. Jungbauer J, Wittmund B, Dietrich S, Angermeyer MC. Subjective burden over 12 months in parents of patients with schizophrenia. Arch Psychiatr Nurs. 2003;17:126-34. 
16. Roick C, Heider D, Bebbington PE, Angermeyer MC, Azorin JM, Brugha TS, et al. Burden on caregivers of people with schizophrenia: comparison between Germany and Britain. Br J Psychiatry. 2007;190:333-8.

17. Grandón $P$, Jenaro C, Lemos S. Primary caregivers of schizophrenia outpatients: burden and predictor variables. Psychiatry Res. 2008;158:335-43.

18. Zendjidjian XY. Challenges in measuring outcomes for caregivers of people with mental health problems. Dialogues Clin Neurosci. 2014;16:159-69.

19. Testart J, Richieri R, Caqueo-Urízar A, Lancon C, Auquier P, Boyer L. Quality of life and other outcome measures in caregivers of patients with schizophrenia. Expert Rev Pharmacoecon Outcomes Res. 2013;13:641-9.

20. Herdman M, Fox-Rushby J, Badia X. 'Equivalence' and the translation and adaptation of health-related quality of life questionnaires. Qual Life Res. 1997;6:237-47

21. Herdman M, Fox-Rushby J, Badia X. A model of equivalence in the cultural adaptation of HRQoL instruments: the universalist approach. Qual Life Res. 1998;7:323-35

22. Regnault A, Hamel JF, Patrick DL. Pooling of cross-cultural PRO data in multinational clinical trials: How much can poor measurement affect statistical power? Qual Life Res. 2015;24:273-7.

23. Murphy HBM. Culture and schizophrenia. In: Al-Issa I, editor. Culture and psychopathology. Baltimore: University Park Press; 1982. p. 221-49.

24. Caqueo-Urízar A, Miranda-Castillo C, Lemos Giráldez S, Lee Maturana SL, Ramírez Pérez M, Mascayano Tapia F. An updated review on burden on caregivers of schizophrenia patients. Psicothema. 2014;26:235-43.

25. van Wijngaarden B, Schene A, Koeter M, Becker T, Knapp M, Knudsen HC, et al. People with schizophrenia in five countries: conceptual similarities and intercultural differences in family caregiving. Schizophr Bull. 2003;29:573-86.

26. Talwar P, Matheiken ST. Caregivers in schizophrenia: A Cross Cultural Perspective. Indian J Psychol Med. 2010;32:29-33.

27. Gonçalves-Pereira $M$, Xavier $M$, van Wijngaarden $B$, Papoila AL, Schene AH, Caldas-de-Almeida JM. Impact of psychosis on Portuguese caregivers: a cross-cultural exploration of burden, distress, positive aspects and clinicalfunctional correlates. Soc Psychiatry Psychiatr Epidemiol. 2013;48:325-35.

28. Li D, Li SM, Tsang HW, Wong AH, Fung KM, Tsui MC, et al. Development and validation of perceived rehabilitation require questionnaires for caregivers of people with schizophrenia. Int J Psychiatry Clin Pract. 2013;17:264-72.

29. Lloyd H, Singh P, Merritt R, Shetty A, Yiend J, Singh S, et al. A comparison of levels of burden in Indian and white parents with a son or daughter with schizophrenia. Int J Soc Psychiatry. 2011:57:300-11.

30. Lloyd H, Singh P, Merritt R, Shetty A, Singh S, Burns T. Sources of parental burden in UK sample of first-generation North Indian Punjabi Sikhs and their white British counterparts. Int J Soc Psychiatry. 2013;59:147-56.

31. Boyer L, Caqueo-Urízar A, Richieri R, Lancon C, Gutiérrez-Maldonado J, Auquier P. Quality of life among caregivers of patients with schizophrenia: a cross-cultural comparison of Chilean and French families. BMC Fam Pract. 2012;13:42

\section{Submit your next manuscript to BioMed Central and take full advantage of:}

- Convenient online submission

- Thorough peer review

- No space constraints or color figure charges

- Immediate publication on acceptance

- Inclusion in PubMed, CAS, Scopus and Google Scholar

- Research which is freely available for redistribution

Submit your manuscript at www.biomedcentral.com/submit 